\title{
HOW MANY LOBSTERS ARE IN THE SEA?
}

\author{
ERNEST ENNS ${ }^{1}$ AND BRUCE SMITH ${ }^{2}$ \\ ${ }^{1}$ Department of Mathematics and Statistics, University of Calgary, T2N 1N4 Canada, ${ }^{2}$ Department of Mathematics \\ and Statistics, Dalhousie University, B3H 3J5 Canada \\ (Accepted January 20, 2000)
}

\begin{abstract}
A lobster fishery must have a reasonable estimate of the number of lobsters within the fishery in order to regulate the annual harvest. Stereological sampling procedures are used to estimate the number of lobsters in a defined region of the sea. From a given point in the fishery, a selective sample in a random direction to the boundary of the fishery is taken. This is repeated to generate a systematic sample from which the total population is estimated.
\end{abstract}

Keywords: lobsters, stereology, stochastic geometry.

\section{INTRODUCTION}

The lobster population off the east coast of Canada is currently an issue of critical interest. There is the possibility of increased fishing pressure due to a Supreme Court ruling on the rights of Native fishers. There has never been a proper assessment of the actual lobster population in the various fisheries. This knowledge is essential to the proper handling of the fishery. This paper hopes to remedy this by suggesting a procedure based on stereological methods to evaluate this lobster population.

A fishery $F$ is a defined area of the sea. It is defined as the region of the sea where the concentration of lobsters is greater than some specified economically viable level, EVL. From any point within the fishery, labelled as $B$ for the Buoy that would be placed there, we will sample at specified distances in a straight line until we pass the EVL. This will be repeated from $B$ to generate a systematic sample from which the number of lobsters in the fishery will be estimated (see Enns and Ehlers, 1988, 1993; Gundersen and Jensen, 1987).

The process of sampling will involve a boat which takes a straight line trajectory from $B$ and samples the sea floor at specified distances from $B$. At a point of sampling, the boat lowers a camera near the sea floor and photographs a circular region within which lobsters may be counted. How close the camera is to the sea floor depends on visibility. A flash would of course be necessary. At a sampling location let $h(r, \theta)=$ the number of lobsters/unit area a distance $r$ in direction $\theta$ from $B$. We continue sampling on a straight line trajectory until $h(r, \theta)<c$ for the $n^{\text {th }}$ time where $n=1,2,3 \ldots$ The EVL defines the concentration $c$.

While on a straight line trajectory from $B$ we will consider two sampling spacings and take $n=1$. These are:

a) Equal spacing:

b) In equal spacing, the boat samples distances $r$ apart until we reach the EVL for the first time.

c) We specify concentration contours and using gradient estimates, sample as close as possible to concentration contours.

\section{THE NUMBER OF LOBSTERS}

$h(r, \theta)$ is the number of lobsters/unit area a distance $r$ in direction $\theta$ from buoy $B$. A Fishery is defined as the region where $h(r, \theta)<c$. The total number of lobsters in the fishery is:

$$
N=\int_{0}^{2 \pi} d \theta \int_{0}^{R(\theta)} r h(r, \theta) d r
$$

$R(\theta)$ is the distance to the EVL measured in direction $\theta$. If $\theta$ is defined as a uniformly distributed random variable on the interval $[0,2 \pi]$, then we may write:

$$
N=2 \pi \varepsilon_{\theta}\left[\int_{0}^{R(\theta)} r h(r, \theta) d r\right]
$$

In order to estimate (2) we will take a systematic sample of $\theta$ of size $m$, denoted by $\theta_{1}, \theta_{2}, \ldots, \theta_{m}$. Then let:

$$
N_{i}=\int_{0}^{R\left(\theta_{i}\right)} r h\left(r, \theta_{i}\right) d r
$$

and $\bar{N}=\sum_{i=1}^{m} N_{i} / m$ is an unbiased estimate of $\mathcal{E}_{\theta}\left[\int_{0}^{R(\theta)} r h(r, \theta) d r\right]$.

The integral (3) must be estimated from sampling information obtained either in case a) or b). In both 
cases, let $r h(r, \theta)=H(r, \theta)$ and $r_{i} h_{i}=H_{i}$ is the data from the $i^{t h}$ sample from $B$. If we define $h(r, \theta)$ to be zero the first time it falls below the EVL, then this provides a sharp demarkation of the extent of the fishery. Hence $H_{0}=H_{n}=0$.
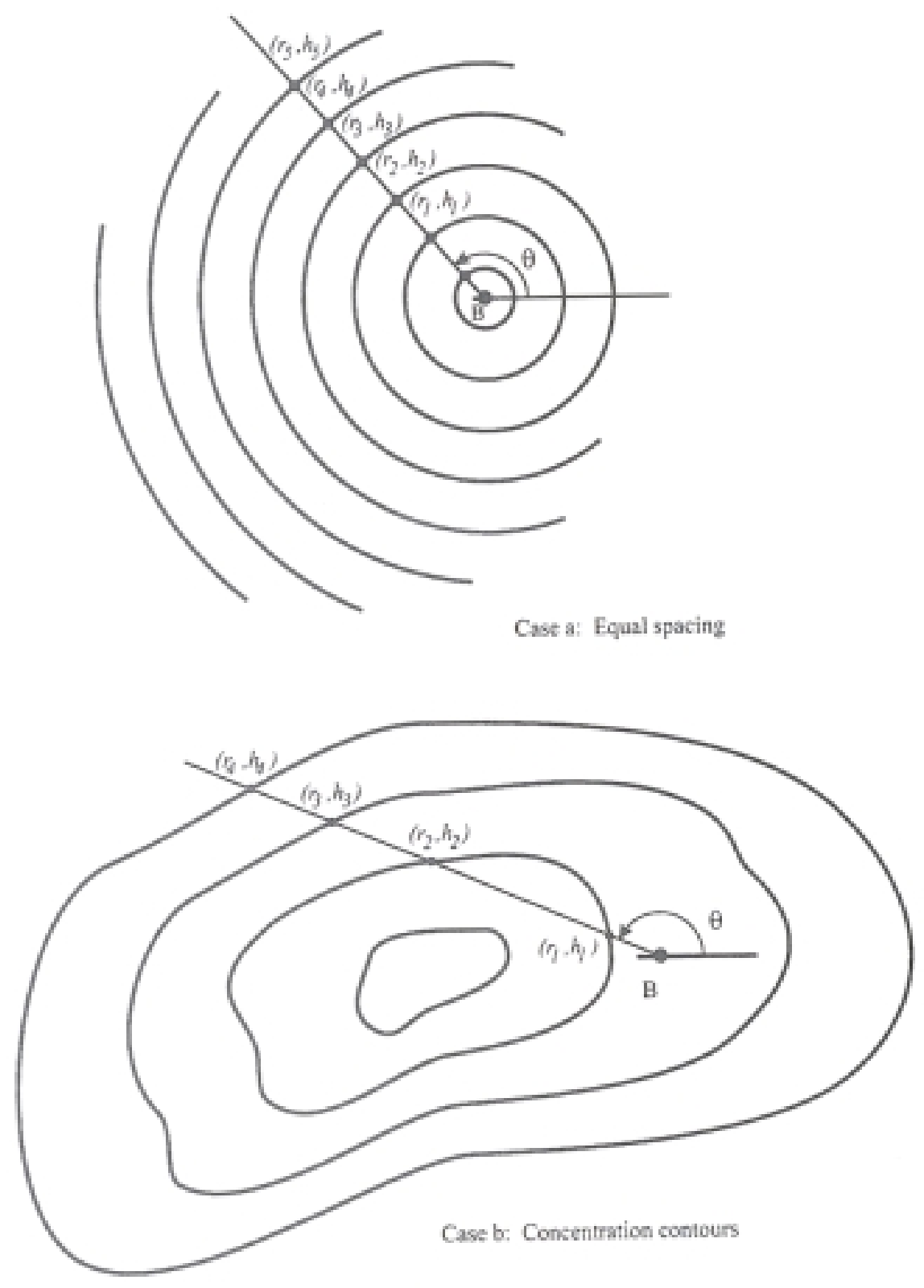

Figure 1

$N_{i}$ as described in (3) may be rewritten as: 


$$
N_{i} \cong \sum_{j=1}^{N}\left(r_{j}-r_{j-1}\right)\left(H_{j}+H_{j-1}\right) / 2 .
$$

This reflects fitting linear segments between contour values of $H(r, \theta)$. (4) can be simplified to:

$$
N_{i} \cong \sum_{j=1}^{n-1} H_{j}\left(r_{j+1}-r_{j-1}\right) / 2
$$

At this point we must consider the two cases:

a) The Equal Spacing Case:

The boat will leave the Buoy $B$ and sample at regular intervals a distance $r$ apart. Then $H_{i}=r_{i} h_{i}=i r h_{i}$ and (5) becomes:

$$
N_{i} \cong r^{2} \sum_{j=1}^{n-1} j h_{j} .
$$

b) The Concentration Contour Case:

In this case we will use the gradient in the sampling direction to estimate how far the boat should travel between sampling points. Specifically $\partial h(r, \theta) / \partial r$ gives us the concentration gradient. Since we only know $h(r, \theta)$ at sampling points, we will estimate

$$
\frac{\partial h(r, \theta)}{\partial r} \text { by }\left(h_{j}-h_{j-1}\right) /\left(r_{j}-r_{j-1}\right) \text { for } j=1, \ldots, \text { n. If we }
$$

wish to estimate the distance to a concentration contour a value $c$, above or below, our present concentration, then the boat should proceed a distance $c\left(\frac{r_{j}-r_{j-1}}{h_{j}-h_{j-1}}\right)$ from the $j^{\text {th }}$ sampling point.

We will not be fortunate enough to always sample on the next concentration contour, hence we can only simplify (5) by using $H_{j}=r_{j} h_{j}$ to obtain:

$$
N_{i} \cong \sum_{j=1}^{n-1} r_{j}\left(r_{j+1}-r_{j-1}\right) h_{j} / 2 \text {. }
$$

\section{SIMULATIONS}

We consider a fishery of lobsters. The actual number of lobsters in the fishery will be 100,000 lobsters for the purpose of the simulation.

The positions of 100,000 lobsters were simulated from a bivariate normal distribution with means zero, variances one, and a covariance of 0.814 . This implies the ratio of the major to minor axes of a confidence ellipse is approximately 10:1.

The local concentration $h(r, \theta)$ was estimated as the number of lobsters within a disk of radius $d r$ centered at $(r, \theta)$. The number of systematically sampled angles was $m$, and the number of points sampled along a ray was $n$. In both the equal spacing and concentration contour case, sampling was begun at the origin. In the equal spacing case the spacing was taken as $4 / n$, and in the concentration contour case, the concentration interval $c$ was taken to be the initial concentration estimate at the origin divided by $n$. The second sample along each ray was taken at radius $4 / n$, after which radial sampling positions were estimated as described above. In each of these schemes, the total area sampled was $A=m n \pi d r^{2}$. With the simulation parameters used, a complete enumeration of the stock would require examination of approximately $9 \pi$ square units.

Twenty independent simulation batches were run at each of $m=6,12,18,24, \quad n=10,20,40, \quad$ and $d r=10,5, .1, .5$. Table 1 lists the estimated mean $\left(\mu_{e}\right)$ and standard deviation $\left(\sigma_{e}\right)$ for the equal spacing case together with the estimated mean $\left(\mu_{c}\right)$ and standard deviation $\left(\sigma_{c}\right)$ for the concentration contour case.

The total area sampled $A$ is reported is in units of $10^{-6}$, which corresponds to the lobster co-ordinates being measured in kilometers, and the radius of the sampling region being measured in metres.

The sampling actually carried out by the Department of Fisheries and Oceans, Canada, is based on occasional trips with lobster fishers, and observations of catch. At present, the department samples about 1 in 6000 boat trips (personal communication, DFO). The lobster density is thought to be in the hundreds or thousands per square kilometer, and the fishing region in many parts of the east coast of Canada, extends up to several hundred kilometers offshore in areas where the continental shelf is wide. Physical sampling devices are sometimes used which have a field of vision of approximatley 30 metres. Given these numbers and the units of the simulation, it is perhaps resonable to consider one of the small values of $d r$ as being plausible. See (Chadwick, 1998) and (Lanteigne et al., 1998).

In these simulations the equal spacing method has little bias while the equal concentration method is biased downwards. In contrast to this, the variability of the equal concentration method is almost always lower than the equal spacing method.

We conjecture that the bias in the equal concentration method is due to inaccurate estimates of the derivative, leading to inaccurate estimates of the next concentration contour. The inaccuracy in the derivative stems from the fact that, at many points, the estimate of $h(r, \theta)$ is based on only a few lobsters contained in the sampling region. 


\begin{tabular}{|c|c|c|c|c|c|c|c|}
\hline$m$ & $n$ & $d r$ & $A$ & He & $\sigma_{t}$ & $\mu_{e}$ & $\sigma_{c}$ \\
\hline 6 & 10 & 10.00 & 0.01885 & 102293 & 22333 & 63063 & 12749 \\
\hline 6 & 10 & 5.00 & 0.00471 & 91307 & 31125 & 57201 & 17673 \\
\hline 6 & 10 & 1.00 & 0.00019 & 77333 & 93943 & 40000 & 64455 \\
\hline 6 & 10 & 0.50 & 0.00005 & 138667 & 399260 & 96000 & 342419 \\
\hline 6 & 20 & 10.00 & 0.03770 & 95480 & 14987 & 75793 & 11393 \\
\hline 6 & 20 & 5.00 & 0.00942 & 100667 & 15790 & 81164 & 15569 \\
\hline 6 & 20 & 1.00 & 0.00038 & 106000 & 112513 & 82067 & 97353 \\
\hline 6 & 20 & 0.50 & 0.00009 & 154667 & 216053 & 120000 & 180551 \\
\hline 6 & 40 & 10.00 & 0.07540 & 99945 & 18140 & 85009 & 12990 \\
\hline 6 & 40 & 5.00 & 0.01855 & 97327 & 16720 & 82741 & 16139 \\
\hline 6 & 40 & 1.00 & 0.00075 & 105667 & 46718 & 94273 & 47175 \\
\hline 6 & 40 & 0.30 & & 119 & 156779 & $102 x$ & 140848 \\
\hline 12 & 10 & 10.00 & & & & & 6552 \\
\hline 12 & 10 & 5.00 & 0.00 & 101 & 19 & & 413 \\
\hline 12 & 10 & 1.00 & 0.00038 & 90667 & 101783 & 50200 & 545 \\
\hline 12 & 10 & 0.50 & 0.00009 & 64000 & 135809 & 37867 & 105791 \\
\hline 12 & 20 & 10.00 & 0.07540 & 93983 & 8044 & 75080 & 8711 \\
\hline 12 & 20 & 5.00 & 0.01885 & 97960 & 13988 & & 11404 \\
\hline 12 & 20 & 1.00 & 0.00075 & 93667 & 56464 & & 47977 \\
\hline 12 & 20 & 0.50 & 0.0 & 693 & 105040 & & 146312 \\
\hline 12 & 40 & 10,00 & & 1008 & & & 7207 \\
\hline 12 & 40 & 5.00 & 0.03 & & 106 & & 9940 \\
\hline 12 & 40 & 1.00 & 0.00151 & 1022 & 490 & & 45562 \\
\hline 12 & 40 & 0.50 & 0.000839 & 77687 & 63918 & 69367 & 62734 \\
\hline 18 & 10 & 10.00 & .05655 & 98498 & 8413 & 63639 & 5756 \\
\hline 18 & 10 & 5.00 & 01414 & 94791 & 15219 & 2154 & 14937 \\
\hline 18 & 10 & 1.00 & 0.00057 & 100444 & 97078 & 16 & 78034 \\
\hline 18 & 10 & 0.50 & 0.00014 & 67556 & 167129 & 39111 & 118712 \\
\hline 18 & 20 & 10.00 & 11310 & 101140 & 4621 & 98 & 3503 \\
\hline 18 & 20 & 5.00 & 27 & 73 & 10260 & & 7419 \\
\hline 18 & 20 & 1.00 & 0.00113 & 95111 & 55910 & 61 & 48691 \\
\hline 18 & 20 & 0.50 & .00028 & 90667 & 81957 & 61333 & 66136 \\
\hline 18 & 40 & 10.00 & 0.22619 & 99518 & 4803 & 85263 & 4688 \\
\hline 18 & 40 & 5.00 & 0.05655 & 102056 & 9588 & 7631 & 6553 \\
\hline 18 & 40 & 1.00 & 0.00226 & 100722 & 31999 & 94674 & 39259 \\
\hline 18 & 40 & 0.50 & & 81556 & 72347 & 70114 & 65541 \\
\hline 24 & 10 & 10.00 & & 97313 & 7360 & & 4175 \\
\hline 24 & 10 & 5.00 & 0.01885 & 97040 & 13063 & 58370 & 11881 \\
\hline 24 & 10 & 1.00 & 0.00075 & 78000 & 59357 & 37333 & 42955 \\
\hline 24 & 10 & 0.50 & 0.00019 & 77333 & 95523 & 37333 & 77577 \\
\hline 24 & 20 & 10.00 & 0.15080 & 99485 & 5133 & 77998 & 3768 \\
\hline 24 & 20 & 5.00 & 0.03770 & 99527 & 9375 & 79877 & 6554 \\
\hline 24 & 20 & 1.00 & 0.00151 & 96000 & 43264 & 68421 & 38106 \\
\hline 24 & 20 & 0.50 & & 866667 & 83154 & 62667 & 67448 \\
\hline 24 & 40 & 10.00 & & & 4843 & 83624 & 4191 \\
\hline 24 & 40 & 5.00 & 0.07540 & 99378 & 8922 & 85909 & 6264 \\
\hline 24 & 40 & 1.00 & & 106000 & 27551 & 91813 & 26315 \\
\hline 24 & 40 & 0.50 & 0.00075 & 94333 & 67988 & 80167 & 62860 \\
\hline
\end{tabular}

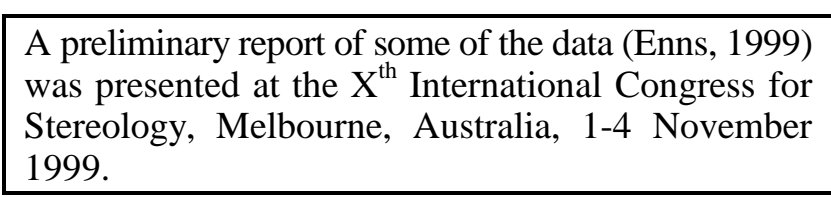

\section{REFERENCES}

Chadwick M (1998). 1998 overview of lobster in the maritimes region. DFO Science Stock Status Report C358,5 pages.

Enns EG, Ehlers PF (1993). Notes on random chords in convex bodies. J Appl Prob 30:889-97.
Enns EG, Ehlers PF (1988). Chords through a convex body generated from within an embedded body. J Appl Prob 25:700-7.

Gundersen HTG, Jensen EB (1987). The efficiency of systematic sampling in Stereology and its prediction. J Microsc 147:229-63.

Lanteigne M, Comeau M, Mallet M, Robichaud G, Savoie F (1998). The american lobster, Homarus americanus, in the southern gulf of St. Lawrence. Canadian Stock Assessment Secretariat Research Document 98/123, 31 pages. 